\title{
Corela
}

Cognition, représentation, langage

HS-1 1 | 2012

RJC Cotexte, contexte, situation

\section{Les rôles du co-texte, du contexte et de la situation dans la lecture en langue étrangère et leur implication pour la lexicographie bilingue}

\section{Sandra Dias Loguercio}

\section{(2) OpenEdition}

Journals

Édition électronique

URL : http://journals.openedition.org/corela/2210

DOI : 10.4000/corela.2210

ISSN : 1638-573X

Éditeur

Cercle linguistique du Centre et de l'Ouest - CerLICO

Référence électronique

Sandra Dias Loguercio, « Les rôles du co-texte, du contexte et de la situation dans la lecture en langue étrangère et leur implication pour la lexicographie bilingue », Corela [En ligne], HS-11 | 2012, mis en ligne le 02 avril 2012, consulté le 30 avril 2019. URL : http://journals.openedition.org/corela/2210 ; DOI : 10.4000/corela.2210

Ce document a été généré automatiquement le 30 avril 2019

\section{(c) (1) (3)}

Corela - cognition, représentation, langage est mis à disposition selon les termes de la licence Creative Commons Attribution - Pas d'Utilisation Commerciale - Partage dans les Mêmes Conditions 4.0 International. 


\title{
Les rôles du co-texte, du contexte et de la situation dans la lecture en langue étrangère et leur implication pour la lexicographie bilingue
}

\author{
Sandra Dias Loguercio
}

\section{Introduction}

1 Le contexte (et/ou le co-texte) aussi bien que la situation encadrant un acte de lecture jouent un rôle essentiel dans la construction du sens d'un texte par le sujet. Cela est vrai autant quand on lit en langue maternelle qu'en langue étrangère. Pourtant la façon de s'appuyer sur ces indices, et la conscience qu'on en a, diffèrent totalement, surtout lorsqu'on ne maîtrise pas suffisamment la langue étrangère. En classe d'apprentissage de lecture en langue étrangère (les cours dits « instrumentaux » en Amérique latine, tels que le français instrumental ou FI), les apprenants débutants apprennent à lire par l'exercice de lecture lui-même, par lequel on leur fait d'abord prendre conscience de tout ce qui concourt à la compréhension textuelle. Puisqu'ils sont souvent des lecteurs expérimentés dans leur langue maternelle (il s'agit d'étudiants de $3^{\mathrm{e}}$ cycle), il faut leur apprendre à lire différemment qu'en langue maternelle, ce qui se passe de façon plus contrôlée, plus consciente (selon une conception volontariste d'apprentissage ${ }^{1}$ ).

2 Cet apprentissage concerne à la fois des éléments linguistiques et discursifs (le lexique et la grammaire, ainsi que l'organisation textuelle, les marques énonciatives, l'image du texte, etc.) et des éléments extralinguistiques (le but et la motivation de lire, l'expérience lectrice, ainsi que les stratégies de lecture, c'est-à-dire comment le lecteur lit le texte et ce qu'il fait pour le lire). Aux premiers, on associe facilement les notions de " contexte» et de «co-texte », qui se distinguent selon l'approche comme nous allons le voir, quant aux seconds, on ne doute pas que la « situation » en est déterminante. Mais qu'est-ce que ces notions recouvrent-elles vraiment? Et comment interviennent-elles lors de la 
lecture? Nous cernerons ces notions à partir des propositions des didacticiens et des linguistes en matière d'enseignement-apprentissage de la lecture, ainsi qu'à partir d'une approche trouvée dans les études de lexicologie.

3 Lié à l'activité de lecture en langue étrangère, et aussi à cet apprentissage, on trouve toujours un outil d'appui (les enseignants étant pour ou contre son usage) : le dictionnaire bilingue (DB), comme en attestent de nombreuses études sur l'usage dictionnairique. C'est pourquoi nous voudrions, après avoir défini les rôles joués par le contexte, le co-texte et la situation dans la construction du sens, réfléchir sur la place du DB lors de la lecture. Nous essayons de préciser enfin quels seraient ses atouts et ses limites par rapport aux notions abordées, ou en d'autres termes, de quoi un DB peut-il rendre compte.

\section{Les rôles du contexte, du co-texte et de la situation dans la construction du sens}

\subsection{L'approche didactique en lecture : la mise en relief du contexte et de la situation}

D. Coste (1974) entame la réflexion sur la lecture du sens qui va finalement constituer la base de l'approche globale employée en FI. Il distingue d'abord deux types de lectures et deux types au moins d'apprentissage: la lecture indirecte, celle qui passe par l'oralisation, qui s'appuie sur l'oral pour arriver au sens, généralement celle pratiquée lors de l'alphabétisation, et la lecture directe, celle qui attribue directement un sens à l'énoncé écrit, pratiquée, entre autres, en FI. Celle-ci diffère de l'autre du fait qu'elle vise à donner du sens au texte et se réalise :

- -de façon active (c'est l'acte d'un sujet),

- -de façon plus globale que linéaire, plus discontinue que continue,

- -en mettant en œuvre chez le sujet des connaissances et un savoir-faire, ainsi qu'une intention et une orientation de lecture,

bref, en appréhendant le texte au moyen de caractéristiques linguistiques ou intratextuelles et de variables intertextuelles ou extralinguistiques.

5 Il y a donc une série d'éléments qui concourent pour la construction du sens en lecture concernant d'abord le sujet lui-même, ses connaissances, son savoir-faire, son but et une demande e/ou envie de lecture (en classe, au travail, pour le plaisir, etc.), c'est-à-dire la "situation" de lecture (les variables extralinguistiques), et puis les caractéristiques textuelles (linguistiques). Celles-ci, D. Coste le fait remarquer, sont constituées de plusieurs plans dépassant celui des mots. De toute façon le « contexte » ici se confond soit avec le cadre textuel - «La saisie d'un énoncé de plusieurs mots dans un temps [...] montre l'importance décisive d'une construction du sens, d'une inférence due au contexte et d'une maîtrise préalable de la langue» (op.cit. p. 42) - soit avec l'axe syntagmatique lorsqu'il parle de prévisions et facilitations dues à «l'entourage contextuel » (op. cit.) lors de tests de « boucher les trous » dans un texte.

6 F. Cicurel et S. Moirand (1990), partant du même point de vue que D. Coste, s'appuient notamment sur l'analyse de l'approche globale réalisée en classe de français dans les pays latino-américains pour relever ce qui est mis en jeu lors de l'apprentissage de la lecture. Elles signalent, entre autres, le fait que les «situations de lecture » en langue étrangère ne sont souvent pas pareilles que celles que l'on connait en langue maternelle: par 
exemple, concernant la lecture de la presse, il n'y a pas les mêmes attentes, on peut ne pas avoir accès à la diversité des journaux en langue étrangère (comme l'on a en langue maternelle), ainsi qu'on peut ne pas avoir accès à la presse orale, on n'est pas dans le lieu où l'on a produit ces documents, ainsi que l'on peut consommer cette production avec un décalage plus ou moins grand dans le temps. Tout cela entraîne des projets de lecture différents, surtout si l'on considère la lecture en classe (où les « projets » de lecture sont plutôt imposés), et joue un rôle essentiel dans les motivations du lecteur, dans sa « fureur de lire ", selon leurs mots.

7 Dans ce sens, la situation de lecture créée en classe par l'approche globale facilite aussi le transfert de certaines stratégies de lecture. Par le moyen de consignes de repérage des indices (iconiques, spatiaux et temporels, thématiques, énonciatifs, bref concernant des petits segments discursifs) sur le texte et leur mise en rapport avec les fonctions principales du texte (descriptif, argumentatif, narratif, etc.), l'enseignant donne des objectifs précis et immédiats aux apprenants lors de cet apprentissage. Cette manière de procéder leur permet de se rendre compte de mouvements d'identification et d'anticipation mis en jeu lors de la lecture et d'acquérir une "compétence de lecture nouvelle ». L'explicitation orale de leurs hypothèses et par la suite la discussion en classe aide à accélérer l'automatisation de ces procédés et donne aux apprenants de plus en plus confiance pour se lancer dans les textes.

8 Sans négliger les autres facteurs impliqués dans cet apprentissage (comme l'approche de la grammaire), c'est la «situation» ici qui déclenche l'acquisition de compétences de lecture. La notion de "contexte ", qui d'ailleurs n'est presque pas mentionnée par ces auteurs, est associée davantage à l'extratextuel, à des connaissances préalables du lecteur, qui permettraient, par exemple, de saisir l'hétérogénéité anonyme dans les discours, la polyphonie énonciative.

9 Cet accent mis sur le sujet (ses expériences, ses connaissances, ses attentes, ses motivations, etc.) nous le trouvons également chez d'autres auteurs réfléchissant sur l'apprentissage de la lecture. Pour J.C. Beacco (2007), par exemple, il est à privilégier en enseignement de la réception des activités qui focalisent «un accès au sens des utilisateurs/apprenants élémentaires fondé sur des connaissances non linguistiques pour n'aborder qu'ensuite des activités hypothético-déductives fondées sur la connaissances de la langue. » (p. 185).

Selon Coracini (1990), c'est tout d'abord les «données individuelles» qui aident à construire les «données textuelles ». Celles-là comprennent la personnalité du lecteur (ses goûts, ses intérêts...), ses idéologies, ses expériences diverses et ses connaissances (sur le sujet abordé, concernant la pratique de lecture, les compétences discursives et sa capacité d'adaptation à un projet de lecture). C'est-à-dire que la situation individuelle du lecteur l'emporte sur les éléments textuels.

11 C'est donc la recherche du sens appuyée sur la «situation » des apprenants qui est mise en relief, soit concernant plutôt leurs connaissances du monde, leurs buts et leurs projets, soit liée à des facteurs psychologiques et socioculturels les caractérisant, telle que met en évidence le travail de C. Dévelotte (1989).

12 Nous rappelons néanmoins que ces travaux, très éclairants en ce qui concerne la réception de textes en langue étrangère, partent en général du point de vue de l'enseignant, pas du point de vue de l'apprenant. Comme le remarque bien B. Rui (2000) quand elle aborde la question des « stratégies de lecture » : 
«il serait nécessaire [...] d'opérer un réel recentrage sur les apprenants-lecteurs en observant empiriquement quelles stratégies de lecture ils mettent en œuvre et non pas en cherchant à retrouver des stratégies de lecture qui correspondent à des préceptes pédagogiques ou théoriques préconstruits. » (op. cit., p. 8)

Or, les stratégies adoptées par les apprenants lors de la lecture tendent à être étroitement liées à ce que l'enseignant développe en classe où, outre le contenu linguistique, sont transmis des savoirs-faires, des habitudes et des croyances relatifs à une pratique ou connaissance donnée. Pourtant il reste toujours le rôle de l'initiative individuelle jouant également pour l'acquisition d'un savoir et/ou pour le franchissement des étapes lors de la réalisation d'une activité. C'est ainsi que nous constatons à côté des stratégies enseignées et stimulées par les enseignants d'autres stratégies qui viennent s'y ajouter lorsqu'il faut, c'est-à-dire lorsque celles-là ne sont pas suffisantes. Le recours au DB en est une.

\subsection{L'approche cognitive en lecture : l'interaction entre le contexte et les éléments linguistiques}

14 Selon les études cognitives du langage (D. Gaonac'h, 1990 ; Coirier, Gaonac'h et Passerault, 1995), une lecture efficace tient surtout à l'interaction ou à la gestion entre deux niveaux différents: un haut niveau (processus de traitement de l'information contextuelle, thématique, du contenu de référence, de caractéristiques relatives au genre textuel...), c'est-à-dire des indices fondés sur des connaissances préalables à une lecture, et un bas niveau (processus de traitements textuels et linguistiques, tels que la reconnaissance des mots et la compréhension de la syntaxe). Le processus de bas niveau, lié surtout à une activité de décodage et de signification, est automatisé en langue maternelle pour les lecteurs expérimentés. Cela libère de l'attention pour la tâche interprétative de lecture, la construction du sens, bref pour le processus dit de haut niveau. C'est justement cet équilibre qu'on ne trouve pas en lecture en langue étrangère. D'après D. Gaonac'h (1990) :

«...les problèmes de compréhension pour certains lecteurs en langue étrangère ne relèvent pas forcément du déficit de processus de haut niveau, mais peuvent être la répercussion d'un déficit d'automatisation de certains processus de bas niveau. » (D. Gaonac'h, 1990, p 77)

En outre, on a également démontré que certaines compétences supposées jouer un rôle essentiel lors de la lecture ne sont pas utilisées ou ont un rôle moins important en langue étrangère, telle que l'identification des indices relatifs au contexte. Il en va ainsi pour la recherche de Carrell (1983 cité par Coirier, Gaonac'h et Passerault, 1995). En essayant de tester l'importance des indications sur le contenu du texte, sur la «transparence » des items lexicaux (comme facilitateurs d'accès au contenu textuel) et sur la familiarité du lecteur avec la thématique, c'est-à-dire des informations relatives au contexte et/ou appréhendées avant la lecture à proprement parler, Carrell révèle des résultats surprenants. En fait, contrairement aux présupposés didactiques, ces indices n'ont pas d'effets pour la lecture en langue étrangère, au moins pas pour les lecteurs intermédiaires et débutants. Cela se passe justement parce que les automatismes dus aux connaissances linguistiques sont défaillants. Paradoxalement, les indices de haut niveau ne peuvent être 
mis vraiment en œuvre par le lecteur que s'ils sont supportés par une maîtrise linguistique, assurant un fonctionnement des processus de bas niveau.

Ainsi l'enseignement-apprentissage concernant la lecture en langue étrangère, selon l'approche cognitive, devrait donc viser à la fois :

- -l'entraînement à l'utilisation de données contextuelles et de connaissances préalables, transférable mais non pas évidente, comme pratiqué par l'approche globale,

- -et l'automatisation, au-delà de la maîtrise, de la mise en œuvre d'éléments du processus de bas niveau.

17 Nous voyons plus clairement ici l'espace forcément laissé ouvert à l'apprenant pour qu'il s'approprie à sa façon les connaissances visées. C'est justement dans cette «lacune » impossible à combler par l'enseignant que ses stratégies individuelles semblent jouer un rôle essentiel pour sa réussite.

\subsection{L'apport lexicologique : la distinction entre co-texte et contexte}

18 Les perspectives lexicologiques fondées sur l'analyse des unités lexicales mettent souvent l'accent sur des notions opératoires qui ne sont pas toujours prises en compte par d'autres linguistes. Ainsi on y trouve le recours aux notions de "lexème » et "vocable ", et de « co-texte » et « contexte » (cf. F. Cusin-Berche, 2003; M-F. Mortureux, 2007), entre autres. Ces notions relèvent d'une approche linguistique qui vise notamment à la description lexicale faisant le lien entre langue et discours.

Plus précises que la désignation "mot ", celles de lexème et vocable permettent de distinguer, respectivement, l'unité abstraite de la langue, traitée dans un dictionnaire, et l'unité prise en discours. Les lexicographes donc «cherchent à construire, à partir des discours qu'ils observent, des représentations du lexique de la langue » (M-F. Mortureux, 2007, p. 13) en offrant des généralisations sur les unités présentées (les lexèmes), c'est-àdire des virtualités linguistiques.

Autant le contexte que le co-texte jouent un rôle pour la détermination du sémantisme des unités lexicales prises en discours, et les deux à la fois sont à prendre en compte lorsqu'on les convertit en lexèmes. Selon F. Cusin-Berche (2003), le co-texte (l'environnement linguistique) permet de prendre en compte la distribution de l'unité et d'approfondir l'analyse sémantique, en établissement un champ sémantique. Tandis que le contexte, associé aux données cognitives et situationnelles (les implicites en discours partagé ou le cadre référentiel), active un champ notionnel et pourvoit l'unité lexicale de sèmes secondaires ou actualise des sèmes virtuels. Aussi peut-on trouver des énoncés, comme nous le montre F. Cusin-Berche (op. cit., p. 24), où les indices co-textuels sont discordants des indices contextuels (les cas mis en évidence par l'ironie, par exemple, certains énoncés hypocoristiques, etc.). Cette discordance pourtant ne modifie pas le sens linguistique (la signification) des unités, mais son interprétation. Cette situation peut aboutir parfois alors à une nouvelle acception ou à une acception spécifique.

21 Ainsi, si l'on prend le mot «parti » dans les énoncés suivants : «Loyauté, respect et

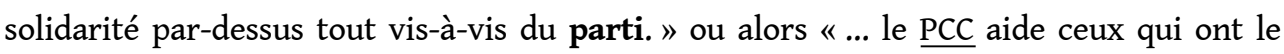
moins de moyens : il subvient aux besoins, [...] il organise des tirages au sort pour des ballons et des bicyclettes lors du jour des enfants. Qui en fait autant? Seulement le parti ! », on ne doute pas qu'il s'agit d'un parti politique, d'ailleurs désigné par le sigle " PCC » (Le monde diplomatique, décembre 2006). C'est le texte et le cadre référentiel qui nous 
révèlent donc que PCC (Premier commando de la capitale) n'est pas un parti comme un autre... mais est une organisation née dans un établissement pénitentiaire à São Paulo (Brésil) dont les « affiliés » ou " partisans » sont tous des criminels.

Le contexte dans ce cas ne crée pas une nouvelle acception ni ne pourvoit l'unité de nouveaux sèmes, mais simplement réactualise des sèmes du mot. Dans une perspective cherchant une construction d'un «signifié unitaire » (cf. Cusin-Berche, 2003, p. 24), on pourrait définir "parti » simplement comme "une association de personnes", où l'on suppose évidemment des règles qui les régissent et des intérêts communs.

Cette approche, allant vers une direction inverse des précédentes, car s'intéressant à l'abstraction et aux virtualités de la langue, fait le lien, dans ce travail, entre texte et entrée lexicale ou traitement lexical tel qu'il est envisagé par la lexicographie. L'apprenant d'une langue étrangère, mais pas seulement l'apprenant, ayant sa lecture, ou plutôt sa construction du sens (son interprétation) entravée par un manque, une lacune, c'est par le lexique qu'il essaiera souvent de trouver les liens manquants. Les unités lexicales fonctionnent à ce titre comme des portes ou des fenêtres dans les textes, permettant un incessant aller-retour tout au long du parcours interprétatif. Car comme nous l'explique F. Rastier (2001, p, 8), « alors que la signification est traditionnellement présentée comme une relation, le sens peut être représenté comme un parcours ». Ce parcours s'appuie à la fois sur des référents externes et des référents internes aux textes ( «l'interréférentialité » sur laquelle R. Galisson, 1983, attire l'attention).

Nous pourrons donc conclure cette partie avec F. Rastier, selon qui « le sens n'est ni dans l'objet (texte), ni dans le sujet (interprète), mais "dans" leur couplage, au sein d'une pratique sociale.» (op.cit., p.125). Ni le contexte ni la situation, tels que définis précédemment, tous seuls, ne contiennent donc les clés pour l'interprétation, la construction du sens.

Les dictionnaires de façon générale, en tant qu'outils de consultations sur la langue et plus spécifiquement sur le lexique, ont sûrement une place dans cette pratique, puisqu'ils sont employés lors de sa réalisation, et leurs contenus (leurs textes) traversent donc, d'une certaine manière, la lecture. Les DB, entre autres, ont leur place dans le parcours de lecture en langue étrangère. Mais laquelle? A quoi vraiment peuvent-ils être utiles ?

\section{Le rapport entre le dictionnaire bilingue et l'apprentissage de la lecture en langue étrangère}

\subsection{L'usage du dictionnaire bilingue en classe de FLE}

De nombreuses recherches sur l'usage du dictionnaire attestent que la tâche pour laquelle il est le plus utilisé est pour la lecture, soit en langue maternelle soit en langue étrangère, et l'information la plus recherchée est la signification des mots (cf. Béjoint, 1981, 2003; Bogaards, 1988, 1995 ; Welker, 2005, 2006, et autres). Il a donc une certaine vocation à informer sur le « sens ».

A ces données, il faut ajouter quelques autres, à savoir (cf. surtout Welker, 2006) :

- le DB est le plus utilisé par des apprenants d'une langue étrangère, surtout pour la compréhension écrite et principalement dans les premières années d'apprentissage ;

- par contre, il est le plus critiqué par ces mêmes usagers ; 
- et enfin il est le plus discrédité par les enseignants de façon générale, son usage n'entrant souvent pas du tout dans le programme pédagogique. s'appuyer sur ses connaissances, sur les indices textuels, inférer donc à partir du « cotexte » et du « contexte » dans une «situation » donnée, les mots qu'il ne connaît pas ou qui ne font pas sens pour lui. Cela évite que le sujet interrompe sa lecture à chaque mot ou qu'il essaie de lire mot à mot, en entravant les mécanismes d'anticipation et la formulation d'hypothèses. De plus, on montre au sujet par là qu'il peut être capable d'accomplir une tâche (un but immédiat) sans comprendre tous les mots d'un texte. C'est une stratégie de lecture qui est enseignée, nécessaire évidemment, et qui fonctionne souvent très bien, comme en attestent les cours de FI. Mais cette stratégie ne peut pas être efficace toujours toute seule.

31 Il reste que les apprenants, notamment les débutants, ne peuvent pas se passer du DB (ou en classe, de l'aide de l'enseignant qui en interdit l'usage), même s'ils le critiquent. Et cela n'est sans doute pas un caprice, mais fait partie de leurs stratégies de lecture (comprises, dans ce cas, non pas comme la façon dont ils lisent, mais plutôt comme ce qu'ils font pour lire). Or comme nous le montrent les recherches cognitives en lecture, les éléments contextuels et situationnels ne peuvent pas être bien mis à profit en vue d'une interprétation globale du texte si l'on n'a pas une maitrise minimum, c'est-à-dire automatisée, des éléments linguistiques (grammaire et lexique). Le texte qui devient un ensemble de «devinettes» pour l'apprenant ne semble pas l'amuser, et peut non seulement le fatiguer et le démotiver mais aussi le tromper énormément. C'est l'autre face de la stratégie de l'inférence.

dictionnaire monolingue, indiqué normalement par les enseignants, n'aide pas beaucoup dans ces cas. Pour les mêmes raisons d'ailleurs: le lecteur qui commence à apprendre une langue, qui n'a donc pas une connaissance linguistique élémentaire, ne peut pas s'en servir. Ce n'est pas par hasard qu'il existe une catégorie de dictionnaires monolingues destinées à des apprenants (les learner's dictionary) ou, plus récemment, des ouvrages bilingues d'apprentissage. Il s'agit de dictionnaires qui répondent aux besoins spécifiques d'apprentissage ou d'un certain niveau de maîtrise de la langue. Ils constituent une sorte de passage pour les apprenants jusqu'au moment où ceux-ci commencent à tirer vraiment profit de grands monolingues. Cependant ce genre de dictionnaire a une nomenclature très limitée et vise notamment à une approche "généraliste » de la langue (écrite et parlée), ce qui ne sert pas non plus dans certaines situations de lecture ou pour la lecture de certains textes. Le vocabulaire qui fait obstacle aux étudiants lorsqu'ils essaient de lire des articles scientifiques ou la presse n'est souvent pas trouvé dans ces ouvrages. 

tant que stratégie de lecture parmi d'autres, dans la mesure où ils y ont recours pendant le processus de compréhension (fait normalement de plusieurs lectures), en s'y appuyant pour accéder à une information linguistique, gagner de la confiance et aussi pour 
apprendre. Le dictionnaire ne remplace pas l'enseignant ni les autres supports d'apprentissage, mais il peut les aider à devenir de plus en plus autonomes, pourvu qu'ils aient appris à l'utiliser. De plus, du fait qu'il présente souvent plusieurs informations réunies sur un même lexème, il peut constituer un outil unique et privilégié d'apprentissage de certains aspects du fonctionnement de la langue à ne pas négliger.

Pour ce qui est des notions traitées ici, nous pourrions avancer les points suivants.

La «situation", en tant qu'aspect lié aux données individuelles et non-linguistiques (connaissances diverses, savoir-faire, familiarité avec des genres textuels, envies, goûts, idéologies...), ne peut évidemment pas faire partie d'un ouvrage, ni déterminer son projet et sa réalisation, car elle est insaisissable et définit l'expérience et le parcours interprétatif de chacun. Pourtant quelques données situationnelles sont à considérer lors de l'élaboration d'un tel ouvrage et vont donc marquer cet ouvrage, à savoir : le niveau linguistique des apprenants et leur intérêt pour la lecture d'articles scientifiques ou diffusés par la presse (les domaines devant être définis, mais rien ne nous empêche de les considérer dans les grandes lignes et d'envisager des ouvrages consacrés aux sciences humaines, aux sciences de la santé, aux sciences juridiques...). Ces données situationnelles détermineront d'abord le corpus, d'où devra être extraite la nomenclature, puis la manière de la présenter (la question de la lemmatisation) et enfin la formulation des articles (le genre définitionnel, les exemples, les explications de toutes sortes, etc.). Celleci doit privilégier plutôt l'explicitation du sens des entrées lexicales que les équivalents en langue-cible, ainsi que les lexies complexes et les difficultés de la langue écrite (caractéristiques du genre article scientifique et de textes de vulgarisation), qui ne peuvent pas être contournées.

Le «contexte», mot fort polysémique, peut concerner, selon l'approche, soit l'extratextuel (englobant donc tous les éléments liés au sujet et aux conditions de l'acte interprétatif), soit le cadre textuel (ou le cadre référentiel), soit encore les éléments linguistiques qui entourent une unité lexicale sur le plan syntagmatique. Puisque tous ces aspects nous intéressent, ils seront définis et nommés différemment, le contexte étant pris, pour nous, dans le deuxième sens, c'est-à-dire en tant que cadre textuel ou référentiel. C'est du genre textuel (l'article scientifique et l'article de vulgarisation) et de domaines de connaissances (notamment ceux de sciences humaines) dont il est question ici. Cela signifie que les unités lexicales et leurs différentes acceptions seront définies à partir de ce contexte (celui du corpus envisagé), ne visant pas l'analyse exhaustive d'emploi des unités. De même des informations pertinentes à l'oralité de la langue, telles que nous les retrouvons souvent dans les DB sous forme d'exemples ou de remarques, ne feront pas objet dans ce cas.

41 Enfin, le « co-texte », soit l'environnement linguistique le plus immédiat des unités, est la notion de toute évidence à être prise en compte par les dictionnaires d'apprentissage. Car s'il peut être évident pour le lecteur maîtrisant la langue, en favorisant par là ses anticipations lors de la lecture, il ne l'est pas pour l'apprenant. C'est donc par les cotextes que le lexicographe peut présenter, refaire, les liens des unités avec les discours où elles s'actualisent. Cela apparaît clairement pour certaines unités, qui ont une aptitude à se combiner avec d'autres, c'est-à-dire pour lesquelles les collocations sont bien marquées, tandis que d'autres ont un éventail plus large d'emploi. Si l'on compare, par exemple, l'usage des mots suivants (respectivement deux adjectifs, deux verbes et deux articulateurs discursifs), on voit que certains se combinent plus régulièrement avec un mot précis ou un type de mot que d'autres d'une même catégorie grammaticale : 
Extraits du Google

\begin{tabular}{|c|c|}
\hline agréable & $\begin{array}{l}\text { C'est agréable de passer une heur... } \\
\text { Une récompense agréable } \\
\text { Paysage très agréable }\end{array}$ \\
\hline péremptoire & $\begin{array}{l}\text { Propos péremptoires } \\
\text { Actualités sur promesses péremptoires } \\
\text { Paternité : gare aux affirmations péremptoires }\end{array}$ \\
\hline regarder & $\begin{array}{l}\text { Sur quel type d'écran ils l'ont regardé } \\
\text { L'Arménie regarde vers l'Europe } \\
\text { Regardons de plus près } \\
\text { Regardez l'actualité }\end{array}$ \\
\hline défrayer & $\begin{array}{l}\text { Sarkozy défraie la chronique sur Dailymotion } \\
\text { Le décolletée d'Angela Merkel défraie la chronique dans la presse } \\
\text { Le blog de DNA qui défraie la chronique } \\
\text { La faillite du géant canadien Nortel a défrayé la chronique }\end{array}$ \\
\hline pourtant & $\begin{array}{l}\text { Il paraît pourtant que l'école est devenue facile } \\
\text { Cette évaluation impossible et pourtant nécessaire } \\
\text { Malgré son air pincé, ..., Isabeau n'a pourtant pas perdu son sens de l'humour }\end{array}$ \\
\hline certes & 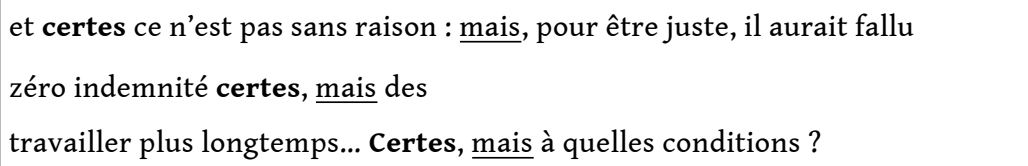 \\
\hline
\end{tabular}

Alors que nous trouvons agréable pour qualifier toutes sortes d'objets ou de situation, péremptoire a un usage beaucoup plus restreint, se limitant à qualifier des noms de l'ordre du discours, de la parole. Il en va de même du verbe défrayer, employé beaucoup plus souvent dans l'expression défrayer la chronique (locution verbale) que tout seul, au contraire évidemment du verbe regarder. Et si l'on étend un peu cette idée, on peut penser également à l'emploi de pourtant et certes. Celui-ci est suivi très souvent de mais, information qui devient pertinente pour saisir sa signification. Le co-texte est donc d'une extrême importance pour l'étudiant-lecteur dans la mesure où, à force de retrouver des combinaisons dans les textes et de les voir telles quelles dans un dictionnaire (qui lui explique et renforce l'information textuelle), il en prend conscience et tend à les automatiser, en augmentant ses possibilités d'inférence.

Il faut souligner finalement que, dans un article de dictionnaire, il y a plusieurs façons de rendre explicite la « contextualisation» des unités. Il n'y a pas de formules, les critères étant à la fois d'ordre linguistiques (objectifs) et pédagogiques (subjectifs) ${ }^{5}$ : les premiers concernent la description de la langue et impliquent d'abord la nature de l'unité traitée, sa fréquence, sa valence, ses combinaisons les plus fréquentes, etc., les deuxièmes concernent le niveau linguistique du public-cible, ses buts, les « critères d'apprenabilité ", 
le rapport entre la pair de langues, entre autres. Très fréquemment, cette contextualisation est fournie par l'information co-textuelle, c'est-à-dire de fragments de discours authentiques, moins souvent voit-on le recours au «contexte», tel que nous l'employons ici.

Dans les DB, cela peut être donné par des exemples, par des éléments différentiateurs (les voisins collocationnels ou des éléments ayant un rapport sémantique avec l'unité traitée, tels que des synonymes, des hyperonymes, des gloses, etc.) donnés dans la langue de départ ou par les valences dans certains cas. Comparons ainsi quelques exemples tirés de différents DB français > portugais ${ }^{6}$ :

\begin{tabular}{|c|c|}
\hline Michaelis & apprendre $v t 1$ aprender, estudar, instruir. 2 ensinar, explicar. \\
\hline Larousse & $\begin{array}{l}\text { apprendre } v t \text { [étudier] aprender; [nouvelle] saber de ; apprendre qqch à } \\
\text { qqn [discipline] ensinar algo a alguém; [nouvelle] informar alguém de algo ; } \\
\text { apprendre à faire qqch aprender a fazer algo. }\end{array}$ \\
\hline $\begin{array}{l}\text { Hachette } \\
\text { Verbo }\end{array}$ & $\begin{array}{l}\text { apprendre } v t r \sim \text { (à faire) aprender (a fazer) ; qch par qn ser informado de } \\
\text { algo por alguém ; (enseigner) ensinar; qch à qn ensinar algo a alguém ; cela } \\
\text { t'apprendra ! Isso há de ensinar-te ! } \diamond \mathbf{s} \sim v p r \mathbf{s}^{\prime} \sim \text { facilement aprender-se } \\
\text { com facilidade }\end{array}$ \\
\hline $\begin{array}{l}\text { Presença/ } \\
\text { Langenscheidt }\end{array}$ & $\begin{array}{l}\text { apprendre leçon: aprender ; nouvelle: saber uma notícia (par qn através de } \\
\text { alguém) ; qc à qn enseigner ensinar qualquer coisa a alguém ; raconter contar } \\
\text { qualquer coisa a alguém ; } \sim \text { à lire aprender a ler }\end{array}$ \\
\hline Michaelis & noyau $\mathrm{nm} 1$ núcleo. 2 centro. 3 Bot caroço. 4 miolo. \\
\hline Larousse & noyau $(\mathrm{pl} \sim \mathrm{x}) \mathrm{nm}$ [de fruit] caroço $m$; [petit groupe] núcleo $m$. \\
\hline $\begin{array}{l}\text { Hachette } \\
\text { Verbo }\end{array}$ & noyau, $p l \sim \mathbf{x} n m$ caroço ; (d'atome) núcleo ; (de la Terre) centro \\
\hline $\begin{array}{l}\text { Presença/ } \\
\text { Langenscheidt }\end{array}$ & $\begin{array}{l}\text { noyau } m(p l \sim x) \text { núcleo } m ; \sim \text { ter [-te] (la) pol introduzir, num determinado } \\
\text { meio, indivíduos encarregados de levar a cabo ações de propaganda ou } \\
\text { subversão }\end{array}$ \\
\hline
\end{tabular}

Les dictionnaires fournissant ces exemples gardent une certaine cohérence dans leur présentation, puisqu'on observe les mêmes principes régissant l'article destiné au verbe apprendre et l'article du nom noyau. Le premier, Michaelis, ne présente aucune sorte de contexte, à l'exception de sa troisième acception du mot noyau, où il indique le domaine. Les autres ouvrages ont recours à des stratégies diversifiées afin d'informer le contexte : d'abord on peut remarquer l'usage d'éléments différentiateurs avant les équivalents proposés en langue cible, ce qui va marquer les différentes acceptions, puis, dans le cas du verbe, on voit le recours à l'indication des valences, enfin, on trouve des exemples dans l'ouvrage Hachette \& Verbo pour le verbe, bien qu'ils soient très limités.

Nous constatons, cependant, par ces extraits de dictionnaires, que le recours au co-texte, aux collocations, telles que nous les trouvons dans les discours, n'est pas privilégié. Nous n'y trouvons pas de fragments de discours. C'est donc à l'usager de refaire, à partir de sa 
lecture, les liens entre les unités en en déduisant leur fonctionnement et leurs sens. Cela peut fonctionner assez bien dans certains cas, mais ne facilite pas l'accès aux significations de l'unité lexicale dans d'autres situations, surtout lorsqu'on a trait à des unités polysémiques, sources potentielles d'ambiguïté. De plus, ce genre d'information abstraite (actancielle), du type «apprendre qqch à qqn » ne nous semble pas favoriser la mémorisation du lexique ${ }^{7}$ à cause de son incomplétude. Si ce procédé peut évidemment aider un usager maîtrisant déjà la langue, il ne nous semble pas être le meilleur choix, s'il vient tout seul, pour un usager débutant qui est en train de l'apprendre.

\section{Considérations finales}

Il ne faut pas perdre de vue finalement que des divisions serrées entre les types de dictionnaires se font de moins en moins, notamment dans le domaine de la lexicographie pédagogique. Aujourd'hui la tendance est de combiner les informations en langue étrangère et en langue maternelle (ou même multilingues) en fusionnant des caractéristiques jusqu'au présent propres aux dictionnaires monolingues et aux dictionnaires bilingues. Grâce aux apports technologiques qui révolutionnent l'accès à l'information dans de tels ouvrages et créent la possibilité d'un stockage (presque) infini de données, les ouvrages en format électronique pourront également répondre sans doute à plusieurs fonctions et satisfaire des publics différents.

Pourtant les questions que nous nous posons en ce qui concerne le rôle du dictionnaire, notamment dans sa fonction de « décodage » pour la lecture et pour l'apprentissage d'une langue étrangère, à partir des notions de co-texte, contexte et situation, sont toujours à considérer lors de son élaboration. Les différentes fonctions d'un même ouvrage sont donc à prévoir séparément, car sa consultation résulte toujours d'une question précise, de besoins linguistiques spécifiques et d'un usager particulier (ayant un niveau linguistique donné, accomplissant une tâche spécifique, etc.). Un dictionnaire qui se propose d'accomplir plusieurs fonctions doit donc répondre simultanément à des situations et des contextes précis, tout en fournissant ou tirant ses informations de co-textes qui rendent compte des différents niveaux de l'information linguistique. En ce qui concerne les dictionnaires pédagogiques ou d'apprentissage, il nous semble que le niveau linguistique de l'apprenant et ses objectifs vis-à-vis de son apprentissage de la langue étrangère constituent des facteurs décisifs pour la conception de ces dictionnaires et ils pourront difficilement satisfaire à la fois un public avancé et un public débutant.

BIBLIOGRAPHIE

BEACCO, J.-C. L'approche par compétences dans l'enseignement des langues. Paris: Didier, 2007.

BÉJOINT, H. « The foreign student's use of monolingual English dictionaries: A study of language

needs and reference skills ». In : Applied Linguistics, v.2, n.3, p. 207-222, 1981. 
BÉJOINT, H. « Vers un dictionnaire bilingue de "médiation" » In : Szende, T. (dir.), Les écarts culturels dans les dictionnaires bilingues. Paris : H. Champion, 2003, pp. 207-221.

BINON, J ; VERLINDE, S ; SELVA, T. « Tendances et innovations récentes en lexicographie pédagogique », De lexicografia : actes del I Symposium International de Lexicografia. Barcelone : Paz BATTANER \& Janet DELESARIS ed., pp. 53-79, 2004

BINON, J. \& VERLINDE, S. «L'enseignement/apprentissage du vocabulaire et la lexicographie pédagogique du français sur objectifs spécifiques (FOS) : le domaine du français des affaires », In : Revue de didactologie des langues-cultures et de lexiculturologie, nº 141, pp. 271-283, 2006.

BOGAARDS, P. « A propos de l'usage du dictionnaire de langue étrangère » In : Cahiers de Lexicologie, $\mathrm{n}^{\circ} 52,1988$, pp. 131-152.

BOGAARDS, P. Le vocabulaire dans l'apprentissage des langues étrangères. Coll. CREDIF, Paris : Didier, 1994

BOGAARDS, P. « Dictionnaires et compréhension écrite » In : Cahiers de Lexicologie, nº 67, 1995-2, pp. 37-53.

CICUREL, F. et MOIRAND, S. « Apprendre à comprendre l'écrit » In : Le Français dans le monde, nº spécial, février-mars 1990, pp. 147-158.

CORACINI, M.J.R.F, « Cursus universitaire de formation au français spécialisée. » In : Le Français dans le monde, nº spécial, août-septembre, 1990, pp. 151-157.

Corier, P. ; GaOnac'H, D ; PASSerault, J.-M. Psycholinguistique textuelle. Paris : Armand Colin, 1996.

coste, D. « Lire le sens » In : Le Français dans le monde, nº 109, décembre 1974, pp. 40-44.

CUSIN-BERCHE, F. Les mots et leurs contextes. Paris : Presses Sorbonne Nouvelle, 2003.

DÉVELOTTE, C. Stratégies de lecture d'un article de presse en langue étrangère. Université Paris III, Cediscor, mémoire de DEA, 1989.

DUDA, W. et al. Zu einer Theorie der zweisprachigen Lexikographie. Überlegungen zu einem neuem russisch-deutschen Wörterbuch. Linguistische Studien. Reihe A. Arbeitsberichte 142 : 1-122, 1986 GALISSON, R. Des mots pour communiquer. Paris : CLE, 1983

GAONAC'H, D. « Lire dans une langue étrangère : approche cognitive » In : Revue Française de Pédagogie, no 93, octobre-novembre 1990, pp. 75-100.

JANITZA, J. « Trois conceptions de l'apprentissage » In : Le Français dans le monde, no 231, févriermars 1990, pp. 38-45.

MORTUREUX, M.-F. La lexicologie entre langue et discours. Paris : Armand Colin, 2006.

RASTIER, F. Arts et sciences du texte. Paris : PUF, 2001

RUI, B. « Exploration de la notion de 'stratégie de lecture' en français langues étrangère et maternelle ». In : Acquisition et interaction en langue étrangère, AILE, no 13, 2000. Disponible sur : http://aile.revues.org/sommaire71.html. Accès le 9/12/2007.

WELKER, H.A. Dicionários. Uma pequena introdução à lexicografia. Brasília : Thesaurus, 2e éd., 2005.

WELKER, H.A. O uso de dicionários. Panorama geral das pesquisas empíricas. Brasília : Thesaurus, 2006.

WELKER, H.A. Panorama geral da lexicografia pedagógica. Brasília : Thesaurus, 2008.

Dicionário Larousse. Mini. Francês-Português, Português-Francês. Larousse, São Paulo, 2005. 
Dictionnaire Portugais. Mini. Français-Portugais, Portugais-Français. Hachette \& Verbo, Paris, 2005.

Langenscheidt. Dicionário Francês-português, Português-francês. Presença, Lisboa, 1998.

Michaelis. Dicionário Escolar. Francês-Português, Português-Francês. Melhoramentos, São Paulo, 2005.

\section{NOTES}

1. Telle que présentée par J. Janitza, 1990.

2. A ce titre, nous ne pouvons pas laisser de mentionner les travaux forts originaux et poussés faits par le Groupe de recherche en lexicographie pédagogique (GRELEP) à Leuven (Katholieke Universiteit), Belgique. Voir notamment S. Verlinde, T. Selva et J. Binon (2004) et S. Verlinde et J. Binon (2006).

3. Même si des innovations technologiques importantes nous donnent forcément aujourd'hui d'autres possibilités d'organisation et élaboration dans le domaine dictionnairique, en élargissant les potentialités lexicographiques en termes de consultation et de réponses, les différentes fonctions d'un dictionnaire sont à envisager séparément.

4. Voir notamment H.A. Welker (2008).

5. P. Bogaards (1994, p. 112) fait la distinction entre "critères linguistiques" et "critères pédagogiques ", tandis que J. Binon et S. Verlinde (2006, p. 274) font la distinction entre « critères objectifs" et "critères subjectifs", mais les notions, telles qu'elles y sont employées, se recoupent à notre avis. Ces critères aident à définir à la fois la sélection de la nomenclature et la formulation des articles.

6. Ces dictionnaires bilingues (français $\leftrightarrow$ portugais) sont tous de petit format (mini ou scolaire), n'excédant pas le nombre de 50000 articles.

7. Idée que nous avançons en tant qu'hypothèse fondée sur les résultats des études cognitives, selon lesquelles l'acquisition d'un vocabulaire se donne par la mise en place des traces mémorielles. Ces traces sont d'autant plus fortes que les expériences sont riches, détaillées et significatives pour le sujet (cf. P. Bogaards, 1994).

\section{RÉSUMÉS}

C'est dans le cadre de l'approche pédagogique mise en œuvre dans les classes de français instrumental que nous nous proposons de cerner les rôles du co-texte, du contexte et de la situation pour la construction du sens lors de la lecture. Nous nous appuyons, tout d'abord, sur trois domaines de recherche afin d'y dégager les différentes définitions de ces notions: tels que la didactique du FLE, les études cognitives en lecture et l'approche lexicologique. Dans un second moment, nous les mettons en rapport avec le rôle joué par le dictionnaire bilingue dans la lecture en langue étrangère, où elles sont donc abordées de la perspective de la lexicographie bilingue et, plus spécifiquement, d'un projet de dictionnaire destiné à la lecture pour des apprenants débutants. Nous voyons, finalement, que ces notions traversent toute l'élaboration d'un tel projet soit en définissant ses critères soit en tant qu'information à fournir par le dictionnaire. 
This study aims to identify co-text, context, and situation roles in the construction of meaning in reading by putting into practice a pedagogical approach during French for Specific Purposes classes. Initially, the research is formulated based on three study areas to extract the different definitions of those concepts, such as: teaching French as a foreign language, cognitive studies in reading, and lexicological approach. Then, a connection is established between those definitions and the role played by a bilingual dictionary while reading in a foreign language, and related themes are addressed under a bilingual lexicography perspective-more specifically, based on a dictionary project intended for novice student use in reading. Eventually, it could be observed that those conceptions permeate the entire preparation of such a project, either by defining its criteria or by representing the information to be provided with the dictionary.

\section{INDEX}

Keywords : co-text, context, situation, french for Specific Purposes, reading in a foreign language, construction of meaning, bilingual lexicography

Mots-clés : co-texte, contexte, situation, français instrumental, lecture en langue étrangère, construction du sens, lexicographie bilingue

\section{AUTEUR}

\section{SANDRA DIAS LOGUERCIO}

Université Paris 3 - Sorbonne Nouvelle 